\title{
Characterization of organic matter in sauce-aroma Chinese liquors by GC-MS and high resolution mass spectrometry
}

\author{
Yue Song ${ }^{1}$, Lingyuan $\mathrm{Xie}^{1, *}$, Jiahao $\mathrm{Li}^{1}$ and Zhouyang Shen ${ }^{1}$ \\ ${ }^{1}$ China University of Petroleum (Beijing), College of Chemical Engineering and Environment, 102249Beijing, China
}

\begin{abstract}
In this study, a total of 25 main compounds of three sauce-aroma Chinese liquors were identified by GC-MS and high-resolution mass spectrometry, and 6 of them (methyl palmitate, ethyl palmitate, methyl linoleate, ethyl linoleate, methyl oleate, and ethyl oleate) were speculated to be characteristic substances of the Maotai series with sauce-aroma type. This study aims to find out the characteristic fingerprint of Chinese liquor and provide a new way for the rapid identification of liquor type and quality. Further experiments are needed to verify the suppose.
\end{abstract}

\section{Introduction}

Chinese liquor, which is one of the oldest distilled spirits in the world, is favored by domestic and foreign consumers because of its exquisite traditional technology, typical style, and excellent product quality. It is quite difficult to analyze due to its rich flavor components and wide content range ${ }^{[1-2]}$. Chinese liquors are composed of three aroma type liquors, including sauce-aroma, strong-aroma and light-aroma types. Among them, sauce-aroma Chinese liquor has a special production process including high temperature Daqu starter, high temperature accumulation, high temperature fermentation, high temperature distillation, long production cycle, long storage time, large dosage of Daqu, and multiple fermentation to get liquor [3], which leads to more complicated composition and unique flavor. Some scholars have systematically reviewed and summarized the research on sauce flavor substances in Chinese liquor of sauce-aroma type, putting forward the conjecture. However, there is still no exact conclusion about the main fragrance of the sauce-aroma type ${ }^{[4]}$. Therefore, it is of guiding significance for liquor brand classification, brewing technology improvement, liquor identification to detect its symbolic organic matter composition.

It has been found that the trace components of sauce-aroma liquors have the following characteristics compared with other flavor types ${ }^{[3,5-7]}$ : (a) high acid content; (b) high aldehyde and ketone content; (c) high n-propanol and heptanol content; (d) high benzaldehyde content and other aromatic compounds; (e) lower ester content but with rich species; (f) pyrazine compounds and furan compounds are the most among all aroma types. Combined with the previous work, three liquors of the Maotai series with different prices were determined by gas chromatography coupled with high resolution mass spectrometry, which is expected to provide a way to quickly identify the series liquor and roughly judge its quality.

In recent years, lots of scholars have focused on the combination of GC-MS, GC-FID, GC-O and other techniques to study the flavor substances of Chinese liquor. However, few reports are available about the use of the high resolution mass spectrometry to study the organic components of Chinese liquor. According to the difference of peak number of high-resolution mass spectrum, we expect to roughly get the difference in the types and numbers of organic matter, which can be used to judge the quality of liquor. In addition, there have been many studies abroad on the analysis of distilled wine and wine by high resolution mass spectrometry (HRMS) combined with GC-MS, GC-FID, GC-O and other methods ${ }^{[8-11]}$, which all mentioned the importance of high resolution mass spectrometry to accurately identify compounds. Generally, a large number of alcohols are often lost in the process of solid phase extraction (SPE), resulting in partial loss of detection outcomes. Liquor samples diluted with methanol were directly injected for high resolution mass spectrometry analysis, which was a good supplement to the results of GC-MS. The quadrupole mass spectrometer (QMF) commonly used in GC-MS has some disadvantages, such as low resolution (unit resolution), interference of isotope, and other $\mathrm{m} / \mathrm{z}$ approximate ions, slow speed and low upper mass limit. While high resolution mass spectrometry can make up for these shortcomings and assist GC-MS to be more accurate. Therefore, the combination of GC-MS and high-resolution mass spectrometry can enhance the accuracy of the experiment and has good feasibility ${ }^{[12]}$.

\footnotetext{
*Corresponding author: 2017011108@student.cup.edu.cn
} 


\section{Materials and methods}

\subsection{Chinese liquor samples}

Three types of commercial soy-sauce aroma liquors were purchased from Kweichow Maotai Distillery (Group) Co., Ltd. in China, which are J-1Maotai Yingbin, 53vol\%; J-2 Laimao, 53vol\%; J-3 Kweichow Daqu in the 1970s, $53 \mathrm{vol} \%$, respectively. The production dates of all samples are within one years.

\subsection{Instruments and reagents}

The organic compounds were separated and identified on an Agilent 7890 gas chromatographer coupled to a Thermo TSQ gas chromatography-mass spectrometry and on a Thermo Orbitrap fusion high resolution mass spectrometer. Other instruments or reagents included methanol (chromatographic purity), acidified water, and solid phase extraction columns (Agilent Technologies).

\subsection{Sample preparation}

Two alternative sample pretreatment methods before analysis were used, their optimizations are described below.

\subsubsection{Direct injection}

Liquors purchased in 2.1 were marked from the lowest price to the highest price as $\mathrm{J}-1, \mathrm{~J}-2$, and $\mathrm{J}-3$, and were diluted to 20,50, 100 times with methanol for later use.

\subsubsection{Solid phase extraction (SPE)}

Methanol and acidified water were added into four SPE columns (1,2,3 and blank control group) to activate SPE columns. $120 \mathrm{ml}$ of liquor sample was taken from each column and was sent to No. 1-3 SPE columns. Acidified water with $\mathrm{pH}=2$ was added to acidify the samples to $\mathrm{pH}=2$, and then the water was removed by nitrogen blowing. Finally, it was washed with $10 \mathrm{ml}$ methanol. The filtrate was collected, heated to evaporate naturally, and concentrated to $4 \mathrm{ml}$. The final extract was directly injected into GC-MS instrumentation; the last extract was directly injected into Orbitrap MS instrumentation after being diluted to 15 times.

\subsection{GC-FID}

Each concentrated fraction ( $1 \mu \mathrm{l}$ )was injected in a splitless mode and analyzed on a $30 \mathrm{~m} \mathrm{HP}-1$ capillary column ( $30 \mathrm{~m}$ x $0.25 \mathrm{~mm}$ i.d. x $0.25 \mu \mathrm{m}$ film thickness; Agilent Technologies, Palo Alto, California, USA); Nitrogen was used as the carrier gas at a constant flow rate of $1 \mathrm{ml} / \mathrm{min}$; The injector temperature was $300{ }^{\circ} \mathrm{C}$. Flame ionization detector is chosen to be the detection of the item.

\subsection{Orbitrap MS}

An Orbitrap mass spectrometer equipped with an ESI source working in positive and negative mode was used in this study. Mass spectra were acquired in profile mode with a setting of 500,000 resolution at the range of $\mathrm{m} / \mathrm{z}$ 50-600. Operation parameters were as follows: spray voltage, 3,000 $\mathrm{V}$ (in positive mode), 2,600 V (in negative mode); ion transfer tube temperature, $300{ }^{\circ} \mathrm{C}$; sheath gas, 6 in positive mode (arbitrary units), 5 in negative mode (arbitrary units); auxiliary gas,2 (arbitrary units).

\subsection{GC-MS}

Each concentrated fraction ( $1 \mu \mathrm{l}$ )was injected in a splitless mode and analyzed on a 60m HP-5 Ms capillary column ( $60 \mathrm{~m}$ x $0.25 \mathrm{~mm}$ i.d. x $0.25 \mu \mathrm{m}$ film thickness; Agilent Technologies, Palo Alto, California, USA); Helium( 99.999\%) was used as the carrier gas at a constant flow rate of $1.5 \mathrm{ml} / \mathrm{min}$; The injector temperature was $300{ }^{\circ} \mathrm{C}$; The temperature program of the oven was as follows: oven temperature was held at $50{ }^{\circ} \mathrm{C}$ for $5 \mathrm{~min}$ at first, then raised to $300{ }^{\circ} \mathrm{C}$ at a rate of $5{ }^{\circ} \mathrm{C} / \mathrm{min}$ and held for $28 \mathrm{~min}$. Mass spectrometry is chosen to be the detection of the item. The MS was operated in electron ionization (EI) mode at $70 \mathrm{eV}$. The identification of organic compounds was conducted in a full scan mode which range was set from 50 to $420 \mathrm{~m} / \mathrm{z}$.

\subsection{Statistical analysis}

The organic compounds were identified by matching with the NIST05 standard spectrum library in Xcalibur software and compounds verified in previous work ${ }^{[13-20]}$.

\section{Results and discussion}

\subsection{Summary of main organic matter components in sauce-aroma liquor}

In this study, the organic components of sauce-aroma liquor were summarized [13-20], and statistics of the molecular formula, chemical structure formula, and relative molecular weight from Chemspider database were used as the reference for qualitative analysis of experimental results.

\subsection{Comparison of the results of direct injection and solid phase extraction}

The result of GC-FID using pre-treatment methods presented in 2.3.2 and the operating conditions in 2.4 was that the chromatogram had only one high peak, which was supposed to be ethanol. It is speculated that the reason for this situation might be the overload caused by too high content of ethanol, and the non-polar column is not suitable for the detection of organic matter in liquor by GC-FID. Previous studies have shown that CP-WAX57CB capillary column could be one ideal column for liquor flavor analysis, which is more suitable 
for gas chromatography with FID as detector for the analysis of high boiling esters ${ }^{[2]}$. Non crosslinked bonded column is more suitable for the analysis of high boiling aromatic components [7]. HP-INNOWAX capillary column has the same characteristics as DB-WAX capillary column, with slight differences in retention characteristics. Compared with DB-WAX capillary column, HP-INNOWAX capillary column has higher temperature resistance and is more suitable for GC-MS analysis. Based on the detection method of GC-MS combined with HRMS, HP-INNOWAX and PEG-20M capillary columns are more suitable for fingerprint analysis of organic matter characteristics of Chinese liquor ${ }^{[12]}$. GC-FID was no longer used for the pre-treatment method of solid phase extraction for detection in this study as the purpose of this study was to find out the characteristic peaks of this series of liquors, instead of the quantitative analysis of specific components. Meanwhile, as this study was a preliminary test of the feasibility of high-resolution mass spectrometry combined with GC-MS for the characteristic fingerprint analysis of liquors, not for the separation of all components, HP-5MS column prepared in the laboratory was firstly used in this study as a test. According to the pre-treatment method presented in 2.3.2 and the operating conditions in 2.5 , the diluent liquor samples with different gradient concentrations were detected by Orbitrap MS (high resolution mass spectrometry). Results include: (a) the response of each sample in the instrument was very small; (b) the peak output of 20 times of dilution was similar with that of 100 times of dilution; (c) no obvious characteristic peak was found in the comparison of three kinds of liquor samples at the same concentration. According to above-mentioned analytical result, combined with the analysis of the organic matter content and a peak output of the original commercial liquor, the pre-treatment method of diluting the original liquor sample should not be used, and the pre-treatment method to concentrate the original liquor sample was considered as valuable in the following experiments. Meanwhile, the high resolution mass spectrometer would be used to detect the organic matter contained in the sample.

For GC-MS detection, the last extracts of SPE were injected directly, of which the specific experimental steps are presented in 2.3.2 and 2.5. For Orbitrap MS detection, the last extracts obtained from SPE 15 times were diluted and injected directly, experimenting followed by 2.4 operating conditions. Experimental results were up to expectations. The analysis of the results was described as follows.

\subsection{Analysis of GC-MS results}

Fig. 1 shows the total ion flow chromatograms of J-1, J-2, and J-3 by GC-MS. A total of 25 compounds of higher abundance were identified among three samples, including alcohols (3-methyl-1-butanol, 2-methyl-1butanol, 1-hexanol, 2-phenyl ethanol), esters (methyl 2-hydroxypropionate, ethyl lactate, ethyl isovalerate, ethyl hexanoate, ethyl 2-hydroxy-4-methyl valerate, ethyl nonanoate, 2-phenyl ethyl acetate, ethyl decanoate, methyl laurate, ethyl dodecanoate, ethyl tetradecanoate, ethyl pentadecanoate, ethyl palmitate, ethyl linoleate, ethyl oleate, methyl palmitate, methyl linoleate, methyl oleate, Diethyl succinate), furans (furfural, 2-acetyl-5methyl furan). It could be observed from the chromatograms that the distribution of the three sample peaks was generally similar, indicating that they could have some of the same organic matter composition. Eight esters with relatively high qualitative abundance (ethyl lactate, ethyl hexanoate, methyl palmitate, ethyl palmitate, methyl linoleate, ethyl linoleate, methyl oleate, and ethyl oleate) were the shared substances of the three samples, among which ethyl lactate and ethyl hexanoate were the esters commonly found in three major aroma types of Chinese liquor. The experimental results in this part confirmed the previous conjecture that liquor sample pretreatment should be concentrated rather than diluted. It was preliminarily speculated that six esters with higher boiling points, namely, methyl palmitate, ethyl palmitate, methyl linoleate, ethyl linoleate, methyl oleate, and ethyl oleate, might be used as the fingerprint information to distinguish sauce-aroma Maotai series liquor with different prices, while the results need to be verified by subsequent experiments.

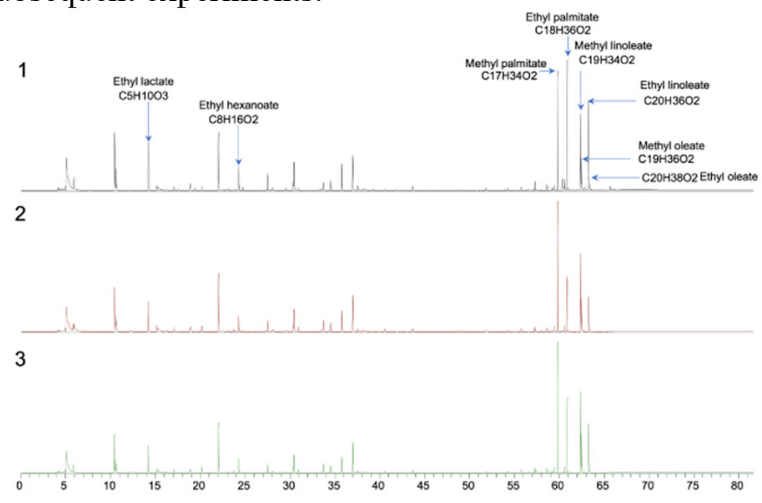

Fig.1 Total ion flow chromatogram of J-1, J-2 and J-3 by GC-MS

\subsection{Analysis of Orbitrap MS results}

\subsubsection{Orbitrap MS (+ESI)}

Fig. 2 shows the Orbitrap MS spectrogram obtained under positive ion mode (ESI). It could be concluded that there are similar components in $\mathrm{J}-1, \mathrm{~J}-2$, and $\mathrm{J}-3$ samples within the range of $250-350 \mathrm{~m} / \mathrm{z}$, while the relative intensities of some components in J-3 samples were significantly higher than that of other samples. According to NIST05 standard library query and GC-MS results, four esters can be identified as common components and J-3 liquor has the highest peak strength, namely methyl palmitate, ethyl palmitate, methyl linoleate, and ethyl linoleate. It was speculated that sauce-aroma Chinese liquors of different quality might have certain characteristics in its range, which could be quickly identified by HRMS. 


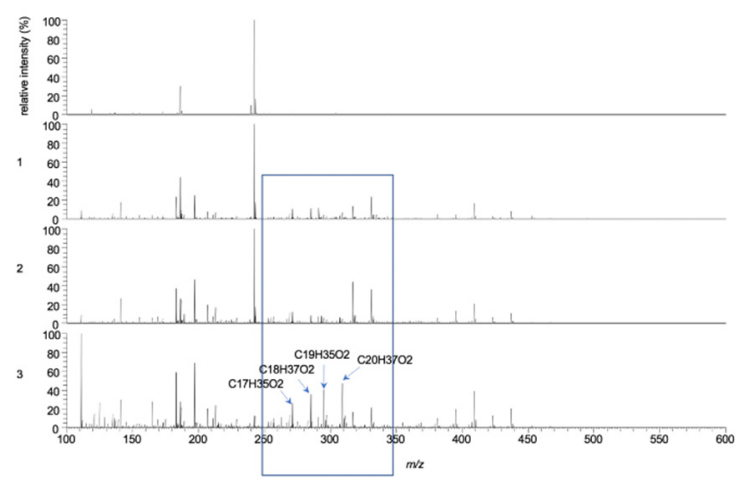

Fig. 2 Mass spectrogram by Orbitrap MS (+ESI)

\subsubsection{Orbitrap MS (-ESI)}

Fig. 3 shows the Orbitrap MS spectrogram obtained under negative ion mode (ESI). It was interesting to note that there were quite similar components with greatly different relative intensities of J-2 and J-3 in the range of $\mathrm{m} / \mathrm{z} 300-450$, where the relative intensities of marked component in liquor sample J-3 were significantly higher than that in liquor sample J-2. We may conjecture that there could be more ions with relatively high nucleus-to-cytoplasm ratio, i.e. more organic matter components with larger molecular weight, in sauce-aroma liquor with higher quality (based on the specified selling price). Therefore, combining the analysis of the results under positive ion mode with that under negative ion mode, we could preliminarily estimate that high resolution mass spectrometry is feasible as a rapid method to identify the quality of the Maotai series of sauce-aroma Chinese liquor..

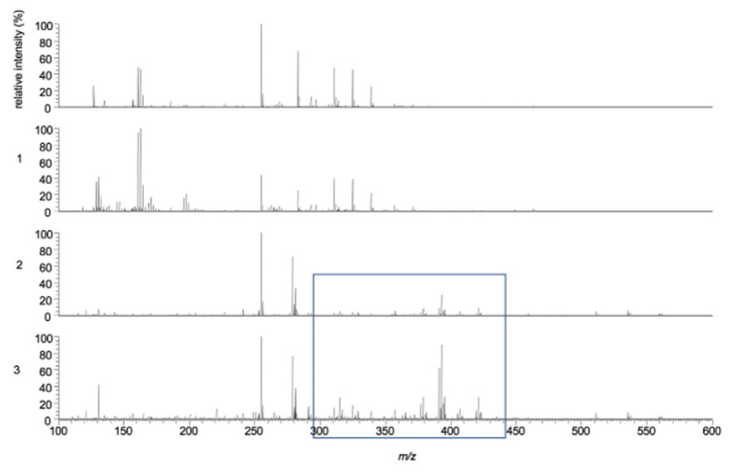

Fig. 3 Mass spectrogram by Orbitrap MS (-ESI)

\subsubsection{N/O heteroatom abundance (+ ESI)}

Fig. 4 shows the heteroatom abundance diagram obtained under positive ion mode (ESI), in which the quantity of nitrogen-containing compounds in sample 3 is significantly less than that in sample 1 and 2 , indicating that most of the ionized compounds in J-3 are compounds containing carbon, hydrogen, and oxygen, and the relative content of other compounds is relatively small. However, the abundance of some nitrogen compounds in sample 2 was the highest among the three samples, which did not show the characteristic difference of sauce-aroma Chinese liquors at different prices. Therefore, the result of N/O heteroatom abundance in this study was not used as the basis for judging liquor quality.

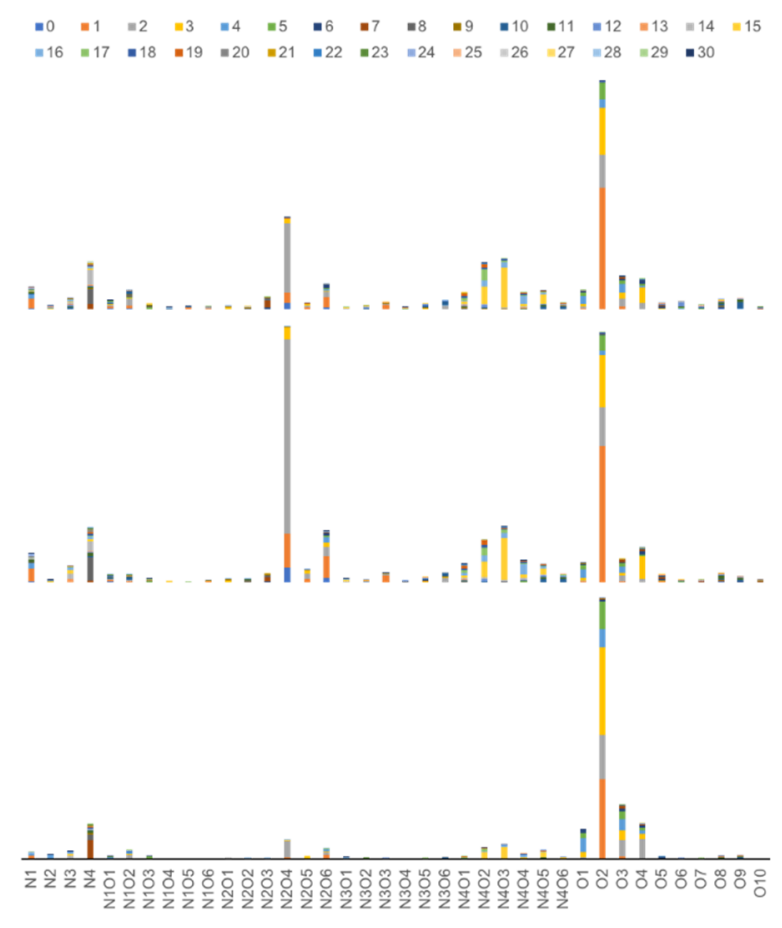

Fig. 4 Abundance diagram of heteroatoms by Orbitrap MS (+ ESI)

\section{Conclusions}

In summary, a total of 25 major aroma compounds of relatively high abundance of three sauce-aroma Maotai series liquor were identified by GC-MS and HRMS. It was speculated that four kinds of esters, i.e. methyl palmitate, ethyl palmitate, methyl linoleate, and ethyl linoleate might be used as a basis for rough identification of the Maotai series with sauce-aroma type by high-resolution mass spectrometry. A few characteristic differences between positive and negative ionization conditions were noticed through the analysis of the experimental results of Orbitrap MS. It was preliminarily verified that high-resolution mass spectrometry could be feasible as a rapid method to identify the quality of sauce-aroma Chinese liquors.

In addition, methyl palmitate, ethyl palmitate, methyl linoleate, ethyl linoleate, methyl oleate, and ethyl oleate were speculated to be the six ester characteristic substances of Maotai series of sauce aroma liquor. Preliminary conclusions need to be verified by vertical and horizontal comparative experiments.

\section{References}

1. Y. F. Shen. Liquor production technology book. China Light Industry Press, (1998).

2. X. Y. Cai. Study on the method of analysis of flavor components in liquor by capillary column direct injection technique. Wine making, (03), 7-10 (2004). 
3. L. Cui. The key technology to form the style and quality of Jiang-flavor liquor is "four high, two long, one big and one more". Brewing, 34(03), (2007).

4. W. L. Fan, Y. Xu. Review and Prospect of the research on sauce flavor substances in Jiang-flavor liquor. Brewing, 39(03), 8-16 (2012).

5. D. Y. Lai. Study on the technological characteristics, flavor characteristics and evaluation points of ten kinds of Chinese flavor liquors. Brewing, (06), 1-6 (2005).

6. J. G. Fu. Study on the relationship between style and technology of Jiang-flavor liquor. Brewing technology, (01), 8-11 (1991).

7. W. L. Fan, Y. Xu. Methodology of research on aroma substances of liquor. Journal of food science and technology, 36(03), 1-10 (2018).

8. T. Rodríguez-Cabo, I. Rodríguez, M. Ramil, A. Silva, R. Cela. Multiclass semi-volatile compounds determination in wine by gas chromatography accurate time-of-flight mass spectrometry, J. Chromatogr. A, 1442, 107-117 (2016).

9. M. Stupak, I. Goodall, M. Tomaniova, J. Pulkrabova, J. Hajslova. A novel approach to assess the quality and authenticity of Scotch Whisky based on gas chromatography coupled to high resolution mass spectrometry, Analytica. Chimica. Acta., 1042, 60-70 (2018).

10. P. Quifer-Rada, A. Vallverdú-Queralt, M. Martínez-Huélamo, G. Chiva-Blanch, O. Jáuregui, R. Estruch, R. Lamuela-Raventós. A comprehensive characterisation of beer polyphenols by high resolution mass spectrometry (LC-ESI-LTQ-Orbitrap-MS), Food Chem., 169, 336-343 (2015).

11. S. Vichi, N. Cortés-Francisco, J. Caixach. Analysis of volatile thiols in alcoholic beverages by simultaneous derivatization/extraction and liquid chromatography-high resolution mass spectrometry, Food Chem., 175, 401-408 (2015).

12. L. Y. Xie, Y. Song, Z. Y. Shen, J. H. Li. Optimization study on fingerprint analysis method of organic components in sauce-flavor liquor based on gas chromatography, Grain Science and Technology and Economy, 45(03), 84-86 (2020).

13. Y. L. Sun, Y. G. Huang, X. C. Zhu. Study on characteristic compounds of light and soy sauce aroma type liquors, Food science, (to be published).

14. H. Y. Shen. Studies on aroma compounds of Chinese soy sauce aroma type liquor, Jiangnan University, (2010).

15. Y. K. Li, G. M. Yang, Q. Z. Li. Analysis of aroma components of Maotai-flavor "Shanzhuang old wine" by $H S-S P M E$ and GC-MS. Brewing Technology, (04), 112-117 (2018).

16. L. Yang, C. Shuang, X. Yan. Analysis of volatile substances in Maotai-flavored salted vegetable flavor defective wine by LLE and HS-SPME Combined with GC-MS. Food and Fermentation Industry, 45(17), 221-226 (2019).

17. X. Z. Sun. Application of CP-WAX capillary column in the analysis of liquor flavor components. Brewing Technology, (12), 87-89 (2005).

18. X. X. Wang. Study on aroma substances and their differences in Maotai-flavor liquor and Luzhou-flavor liquor. Jiangnan University, (01), (2014).

19. X. Zhang, T. Liu, L.P. Wu. Determination of seven phenolic antioxidants and p-hydroxybenzoate preservatives in various foods by high performance liquid chromatography tandem mass spectrometry. Food and Fermentation Industry, 42(11), 206-211 (2016).

20. X. R. Qi, C. Yan, C. H. Song. Analysis of aroma components of three kinds of liquors in Hebei by OAV. Brewing Technology, (09), 95-100 (2018). 